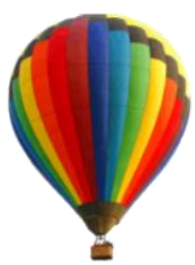

\title{
ANÁLISE DA VALIDADE E CONFIABILIDADE DO MODELO SERVQUAL ADAPTADO AO VAREJO SUPERMERCADISTA
}

\section{ANALYSIS OF VALIDITY AND RELIABILITY OF THE SERVQUAL MODEL ADAPTED TO THE SUPERMARKET RETAIL}

\section{José Wendel dos Santos ${ }^{*}$, Odelsia Leonor Sanchez de Alsina ${ }^{2}, \&$ Luciano Fernandes Monteiro ${ }^{3}$}

${ }^{13}$ Departamento de Engenharia de Produção (DEPRO), Universidade Federal de Sergipe (UFS), Avenida Marechal Rondon, s/n, Bairro Jardim Rosa Elze, CEP 49100-000, São Cristóvão/SE. ${ }^{2}$ Instituto de Tecnologia e Pesquisa (ITP), Universidade Tiradentes (UNIT), Avenida Murilo Dantas, 300, Bairro Farolândia, CEP: 49032490, Aracaju/SE.

1*wendel@email.com² odelsia@uol.com.br ${ }^{3}$ lucianofm@uol.com.br

\section{ARTIGO INFO.}

Recebido em: 03.12.2020

Aprovado em: 14.12.20202

Disponibilizado em: 17.12 .2020

\section{PalaVRas-ChaVe:}

Qualidade em serviços; supermercados; validade; confiabilidade.

\section{KEYWORDS:}

Quality in services; supermarkets; validity; reliability.

\footnotetext{
*Autor Correspondente: Santos, J. W.
}

\section{RESUMO}

Os instrumentos utilizados para mensurar a qualidade dos serviços prestados por determinada organização devem apresentar propriedades favoráveis de confiabilidade e validade, para que justifiquem a confiança que é depositada nos resultados que produzem. Nesse sentido, este estudo objetivou apresentar a aplicabilidade da análise estatística para investigar as propriedades de validade e confiabilidade do questionário aplicado para avaliar a qualidade dos serviços prestados por um supermercado localizado na cidade de Aracaju/SE. Com base nos escores dos 385 consumidores aplicouse a Regressão Linear Múltipla para verificar a validade do questionário, enquanto $o$ alfa de Cronbach para analisar a confiabilidade do mesmo. Nesta análise, observou-se que $78,8 \%$ das variações do grau de expectativa e $80,3 \%$ das variações do grau de desempenho relatados pelos consumidores podem ser explicados pela variação dos escores obtidos nas dimensões avaliadas. Para a mensuração da expectativa, o valor do alfa foi de 0,87 e para mensuração do desempenho foi de 0,86. Esses valores foram considerados satisfatórios, pois os coeficientes encontraram-se acima dos limiares preconizados na literatura especializada. Dessa forma, sugere-se que o questionário empregado na avaliação da qualidade dos serviços do supermercado apresentou-se consistente e preciso.

\section{ABSTRACT}

The instruments used to measure the quality of services provided by a given organization must have favorable properties of reliability and validity, to justify the confidence that is placed in the results they produce. In this sense, this study aimed to present the applicability of the statistical analysis to investigate the validity and reliability properties of the questionnaire provided to evaluate the quality of the services provided by a supermarket located in the city of Aracaju/SE. Based on the scores of 385 consumers, Multiple Linear Regression was applied to verify the validity of the questionnaire, while Cronbach's alpha to analyze the reliability of the questionnaire. In this analysis, it was observed that $78.8 \%$ of the variations in the degree of expectation and $80.3 \%$ of the variations in the degree of performance reported by consumers can be explained by the variation of the scores obtained in the evaluated dimensions. For the measurement of expectation, the alpha value was 0.87 and for the measurement of performance, it was 0.86. These values were considered satisfactory since the previous coefficients were above the thresholds recommended in the specialized literature. Thus, it is suggested that the questionnaire used to assess the quality of services in the supermarket was consistent and accurate. 
Citação (APA): Santos, J. W. dos, Alsina, O. L. S. de, \& Monteiro, L. F. (2020). Análise da validade e confiabilidade do modelo Servqual adaptado ao varejo supermercadista. Brazilian Journal of Production Engineering, 6(7), 120-132.

\section{INTRODUÇÃO}

O setor de serviços, sobretudo na última década, tem ganho destaque, uma vez que, constantemente novos serviços são lançados para satisfazer as necessidades existentes. Por estar em constante crescimento, fomenta-se a concorrência entre as organizações, fazendo com que busquem sua posição de mercado vis-à-vis com suas competidoras (Roy, Sreejesh, \& Bhatia, 2019). Entretanto, a maioria das empresas continuam somando esforços visando ao aumento de produtividade e à redução de custos, deixando a prestação de serviço sem a devida atenção.

De acordo com Kotler \& Armstrong (1998), o fornecimento de serviços de qualidade é uma das principais maneiras de uma empresa se diferenciar no mercado, pois possibilita uma maior aquisição e retenção de clientes (Álvarez-García, et al., 2019; Ferguson, \& Zawacki, 1993). Portanto, consubstanciado nesse contexto, admite-se que o nível de qualidade na prestação de serviços torna-se imprescindível dentro de uma organização, representando um fator-chave para o seu sucesso e sua consequente sobrevivência no mercado.

Na visão de Schneider \& White (2004), o tema qualidade em serviços é um dos conceitos mais discutidos na literatura, principalmente devido a não existência de um consenso sobre sua definição ou ao modo mais adequado de realizar sua medição. Assim, sua avaliação tornase subjetiva. Pode-se atribuir à essa subjetividade fatores que estão diretamente atrelados às percepções intrínsecas de cada indivíduo no processo avaliativo. Essa percepção individual pode ser proporcional às suas experiências anteriores e, às vezes, mesmo de fatores efêmeros e temporários, como necessidades e expectativas específicas.

Por esta razão, muitos pesquisadores têm contribuído no desenvolvimento de métodos para realizar a mensuração da qualidade em serviços. Dentre os modelos propostos para este fim, o Servqual se sobressai. Segundo Ko \& Chou (2020), o modelo Servqual é usado para diagnosticar os pontos fortes e fracos da qualidade do serviço, e é amplamente considerado como o instrumento mais abrangente para esta avaliação. Operacionalmente, o modelo leva em consideração as expectativas dos clientes em relação a um determinado serviço frente à percepção de qualidade do serviço recebido, por meio de atributos agrupados em cinco fatores: aspectos tangíveis, confiabilidade, presteza, segurança e empatia. A partir de cada par de assertivas, pode-se identificar o nível de expectativa e percepção dos clientes em relação ao serviço prestado por determinada empresa, e assim, obtém-se um índice que pode ser positivo ou negativo; quanto maior for o índice, maior será a superioridade do serviço e satisfação do cliente (Roy, Sreejesh, \& Bhatia, 2019; Santos, 2016; Santos, Gomes, \& Vasconcelos, 2017; Parasuraman, Zeithaml, \& Berry, 1988).

Verifica-se, todavia, que por ser um modelo universal passível de aplicação em todos os tipos de organização e setores de serviços, faz-se necessária a introdução de adaptações na redação de alguns itens, para que estes reflitam a realidade de cada investigação (Ghotbabadi, Feiz, \& Baharun, 2015; Freitas, \& Freitas Neto, 2017; Freitas, Bolsanello, \& Viana, 2008). De acordo com Santos (2016), em estudos conduzidos com o modelo Servqual observou-se pouca aderência estatística em algumas tipologias de serviços, principalmente em relação aos fatores

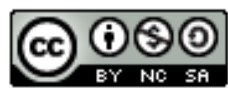


Citação (APA): Santos, J. W. dos, Alsina, O. L. S. de, \& Monteiro, L. F. (2020). Análise da validade e confiabilidade do modelo Servqual adaptado ao varejo supermercadista. Brazilian Journal of Production Engineering, 6(7), 120-132.

essencialmente necessários à realidade do tipo de serviço em contraposição à estrutura adaptada do modelo.

Assim sendo, torna-se necessária a validação e avaliação do grau de confiabilidade do modelo adaptado, buscando investigar se o mesmo possui consistência para medir o que foi determinado a medir quando aplicado repetidas vezes. Nesta perspectiva, propõe-se neste estudo analisar a validade e confiabilidade do modelo Servqual operacionalizado em um supermercado da região metropolitana de Aracaju/SE.

\section{MARCO TEÓRICO}

\section{VALIDADE E CONFIABILIDADE DE QUESTIONÁRIOS}

De acordo com Freitas \& Rodrigues (2005), em toda pesquisa de opinião, a captação de informações dos avaliadores consiste em uma das etapas de maior importância no processo de avaliação. Para eles, essa etapa deve ser realizada através do emprego de um instrumento de medição eficaz e preciso, pois caso contrário, as informações obtidas podem não ser representativas da percepção dos avaliadores. Ou seja, os questionários precisam apresentar propriedades favoráveis de confiabilidade e validade.

Em linhas gerais, a confiabilidade está relacionada à homogeneidade das respostas dos avaliadores, enquanto a validade está associada ao grau de certeza que se tem sobre o conceito medido. A validade é o grau com que os indicadores unidimensionais da escala medem com consistência as dimensões que foram designadas para medir. Já a confiabilidade é o grau no qual o instrumento é consistente em medir o que foi determinado a medir, obtendo os mesmos resultados se aplicado repetidas vezes (Adeniran, 2019; Bolarinwa, 2015).

Malhotra (2012), ressalta, entretanto, que o grau de confiabilidade e de validade estão atrelados a possíveis erros aleatórios, que para ele, não é constante, e representa fatores transitórios que influenciam no escore observados de maneiras diferentes cada vez que se faz a mensuração.

Segundo Freitas \& Rodrigues (2005), quando a variância associada a erros aleatórios diminui, a variância dos valores observados tende a se aproximar da variância dos valores verdadeiros, representando maior confiabilidade ao questionário. Consubstancialmente, a confiabilidade e a validade refletem o quanto os valores observados estão correlacionados aos valores verdadeiros (Hora, Monteiro, \& Arica, 2010).

Entre as modelagens matemáticas mais elaboradas para analisar o comportamento do erro aleatório, o coeficiente alfa de Cronbach e regressão linear múltipla são os mais utilizados. A análise da regressão linear múltipla é uma técnica largamente empregada no processo de validação, por permitir fazer inferências estatísticas precisas, enquanto o coeficiente alfa de Cronbach nos fornece estimativas do grau de confiabilidade.

\section{Regressão Linear MúltiPla}

A análise de regressão linear é uma técnica estatística amplamente usada em aplicações práticas (Jan, \& Shieh, 2019). Nessa análise, tem-se o interesse em avaliar o relacionamento entre um conjunto de variáveis independentes $(X i)$ com uma única variável dependente $(Y), i$ $=1,2, \ldots, p$. Quanto mais significativo for a variável dependente, tanto mais se poderá afirmar 

adaptado ao varejo supermercadista. Brazilian Journal of Production Engineering, 6(7), 120-132.

que alguns fatores afetam mais o comportamento de uma variável do que outros. Portanto, um possível modelo para avaliar essa relação pode ser dado pela Equação 1.

$$
Y_{i}=\alpha+\beta_{1} X_{I}+\beta_{2} X_{2}+\ldots+\beta_{p} X_{p}+\varepsilon
$$

Em que, os coeficientes $\beta p$ indicam o peso da variável independente $(X i)$ sobre a variável dependente $(Y i)$ (Hair, et al., 1998). O termo $\alpha$ é uma constante para adequação de escala. Em termos práticos, quando todas as variáveis independentes assumem o valor 0 , tem-se $Y=\alpha . \mathrm{O}$ termo $\varepsilon$ ou resíduo, representa a diferença entre o valor efetivamente observado e o valor estimado (Bittencourt, et al., 2011). Assume-se que esses erros são independentes e seguem distribuição normal com média zero e variância desconhecida.

A Equação 1 representa o modelo chamado de regressão linear múltipla e seu resultado é um único valor que representa uma combinação do conjunto inteiro de variáveis que melhor atinge o objetivo da análise multivariada específica.

Em regressão linear múltipla recomenda-se utilizar o coeficiente de determinação ajustado $\left(R^{2}\right)$, com $R^{2} \in[0,1]$, para que a habilidade preditiva seja aferida. Esse coeficiente indica o percentual da variabilidade total de $Y$ associada com o uso do conjunto de variáveis $\left(X_{1}\right.$, $\left.X_{2}, \ldots, X_{p}\right)$. Ele pode variar de 0 a 1 . Quando o coeficiente de correlação é maior que 0 , as duas variáveis são positivamente correlacionadas, em contrapartida quando é menor que 0 , as duas variáveis são negativamente correlacionadas. Assim, um alto valor de $R^{2}$ implica que o modelo ajustado se presta para se fazer inferências precisas.

\section{COEFICIENTE ALFA DE CRONBACH}

Segundo Taber (2018), o alfa de Cronbach $(\alpha)$ é um método amplamente utilizado para demonstrar que os questionários elaborados ou adaptados são adequados para o propósito. Esse coeficiente foi proposto por Cronbach (1951) para quantificar a confiabilidade de questionários a partir da análise do perfil das respostas dadas pelos avaliadores. $\mathrm{O}$ alfa de Cronbach é obtido a partir da variância dos componentes individuais e da variância da soma dos componentes de cada avaliado, buscando investigar as possíveis relações entre os itens que compõe o questionário (Cronbach, 1951). A fórmula proposta por Cronbach é dada pela Equação 2.

$$
\sigma=\left[\frac{k}{k-1}\right] \cdot\left[1-\frac{\sum_{i=1}^{k} S i^{2}}{S t^{2}}\right]
$$

Em que: $k$ refere-se ao número de itens que compõe o questionário; $S_{i}{ }^{2}$ representa a variância de cada item; $S_{t}{ }^{2}$ refere-se à variância total do questionário.

Nota-se na Tabela 1, que cada coluna indica um item, cada linha indica um avaliador, e o encontro entre um item e um avaliador, indica a respectiva resposta para o item avaliado, com base na escala especificada. Sucintamente, o coeficiente pode ser calculado em duas etapas: primeiramente é necessário calcular a variância de cada coluna $i$, em seguida somar todas estas variâncias. Na segunda etapa deve-se obter a soma total dos julgamentos de cada avaliador, em seguida calcular a variância destas somas (Cronbach, 2004).

\section{@ $\odot \Theta \odot$}


Citação (APA): Santos, J. W. dos, Alsina, O. L. S. de, \& Monteiro, L. F. (2020). Análise da validade e confiabilidade do modelo Servqual adaptado ao varejo supermercadista. Brazilian Journal of Production Engineering, 6(7), 120-132.

Tabela 1. Matriz de julgamentos dos $n$ avaliadores à luz dos $k$ itens do questionário

\begin{tabular}{cccccccc}
\hline \multicolumn{7}{c}{ Itens } \\
\hline Avaliadores & $\mathbf{1}$ & $\mathbf{2}$ & $\ldots$ & $\boldsymbol{i}$ & $\ldots$ & $\boldsymbol{k}$ & Soma ou Total \\
\hline $\mathbf{1}$ & $X_{11}$ & $X_{12}$ & $\ldots$ & $X_{1 i}$ & $\ldots$ & $X_{1 k}$ & $\boldsymbol{X}_{\boldsymbol{I}}$ \\
$\mathbf{2}$ & $X_{21}$ & $X_{22}$ & $\ldots$ & $X_{21}$ & $\ldots$ & $X_{2 k}$ & $\boldsymbol{X}_{2}$ \\
$\vdots$ & $\vdots$ & $\vdots$ & $\vdots$ & $\vdots$ & $\vdots$ & $\vdots$ & $\vdots$ \\
$\mathbf{p}$ & $X_{p 1}$ & $X_{p 2}$ & $\ldots$ & $X_{p i}$ & $\ldots$ & $X_{p k}$ & $\boldsymbol{X}_{\boldsymbol{p}}$ \\
$\vdots$ & $\vdots$ & $\vdots$ & $\vdots$ & $\vdots$ & $\vdots$ & $\vdots$ & $\vdots$ \\
$\mathbf{n}$ & $X_{n 1}$ & $X_{n 2}$ & $\ldots$ & $X_{n i}$ & $\ldots$ & $X_{n k}$ & $\boldsymbol{X}_{\boldsymbol{n}}$ \\
\hline
\end{tabular}

Fonte: Cronbach (2004).

Para se ter um valor adimensional para representar a isenção dos erros aleatórios das observações, divide-se o estimador pela variabilidade total do questionário. Dessa forma, o alfa de Cronbach é uma propriedade inerente do padrão de resposta dos respondentes, não uma característica da escala por si só; ou seja, o valor de alfa sofre mudanças segundo a população na qual se aplica a escala (Streiner, 2003; Cronbach, 2004; Hora, Monteiro, \& Arica, 2010).

A literatura sugere o valor mínimo aceitável para o alfa de 0,70, cujos valores inferiores resultam em consistência interna baixa. Em relação ao valor máximo, ressalta-se que valores superiores a 0,90 indicam redundância, ou seja, inúmeros itens estão medindo exatamente o mesmo atributo; portanto, os itens redundantes devem ser eliminados. Dessa forma, recomenda-se valores de alfa entre 0,80 e 0,90 (Almeida, Santos, \& Costa, 2010; Hair, et al., 2019; Hora, Monteiro, \& Arica, 2010).

De acordo com Hora, Monteiro \& Arica (2010), o uso do coeficiente alfa de Cronbach deve se submeter a alguns pressupostos:

a) O questionário deve estar dividido e agrupado em "dimensões", isto é, itens que avaliam uma mesma característica;

b) O questionário deve ser aplicado a uma amostra significativa e heterogênea da população;

c) A escala já deve estar validada, ou seja, certificar-se de que o instrumento usado na medição está realmente medindo aquilo que ele se propõe a medir.

Almeida, Santos \& Costa (2010) destacam que, o valor de alfa é afetado pelo número de itens que compõem o questionário. Ou seja, à medida que se aumenta o número de itens, aumentase a variância de tal forma que se obtém um valor superestimado da consistência interna da escala. Do mesmo modo, deve-se considerar que o valor do alfa de Cronbach pode ser superestimado caso não seja considerado o tamanho da amostra: quanto maior o número de respondentes, maior é a variância esperada (Hair, et al., 2019; Krus, \& Helmstadter, 1993; Bland, \& Altman, 1997).

\section{MÉTOdOS DA PESQUISA}

Trata-se de um estudo exploratório, de corte transversal e abordagem quantitativa. O estudo foi conduzido em um supermercado de médio porte localizado na região metropolitana da cidade de Aracaju/SE. 

adaptado ao varejo supermercadista. Brazilian Journal of Production Engineering, 6(7), 120-132.

O supermercado pesquisado funciona de segunda-feira a sábado, das $8 \mathrm{~h}$ às $23 \mathrm{~h}$ e possui uma área total de $1.154 \mathrm{~m}^{2}$, sendo $993 \mathrm{~m}^{2}$ destinados a vendas. Possui 56 funcionários que trabalham 8 horas/dia, em regime de revezamento. Em relação ao sortimento de produtos, o supermercado possui 14 mil itens cadastrados na base de dados, no entanto, encontravam-se apenas 11 mil itens disponíveis para venda. Além disso, possui 16 gôndolas/prateleiras e 9 checkouts, os quais operam com potencial máximo apenas na sexta-feira e sábado, enquanto de segunda-feira à quinta-feira geralmente operam 5 a 7.

A população desta pesquisa foi composta por 10.437 consumidores cadastrados na base de dados do supermercado. O tamanho mínimo da amostra foi obtido a partir do método desenvolvido por Barbetta (2002), sendo considerado um erro máximo tolerável para a pesquisa de $5 \%$ e um nível de confiança de $95 \%$. Assim, desta população, foi extraída uma amostragem probabilística de 385 consumidores para garantir a robustez dos resultados observados.

A coleta dos dados foi realizada in loco nos três turnos de funcionamento do supermercado entre os meses de dezembro de 2019 e fevereiro de 2020. Por questões operacionais, os consumidores foram abordados uma única vez após a efetivação de todo o processo de compra. $\mathrm{Na}$ abordagem, foram instruídos sobre a natureza voluntária do estudo, e foi esclarecida a utilização das informações e a garantia do sigilo das respostas.

Em relação a concepção do questionário, baseou-se na metodologia desenvolvida por Parasuraman, et al., (1990). Em síntese, a primeira parte do modelo constitui-se de vinte e dois itens desenvolvidos para medir o nível expectável - ou ideal - do serviço de uma determinada empresa. Já a segunda parte (novamente os mesmos vinte e dois itens) mede a percepção - esta é a real, objetiva - do cliente sobre o serviço oferecido por uma determinada empresa. Então a qualidade do serviço é avaliada subtraindo-se a qualidade expectável da qualidade percebida na determinada empresa.

O questionário adaptado utilizado neste estudo contém lacunas de respostas para Expectativa (E) e para Desempenho (D), nas quais são utilizadas escalas do tipo Likert com cinco pontos cada. Essas escalas buscam captar o grau de concordância e discordância dos usuários em relação às sentenças afirmativas relativas aos critérios considerados. Assim, para avaliar o grau de expectativa, os avaliadores posicionaram-se entre baixíssima relevância (1) e altíssima relevância (5). Para mensurar o grau de desempenho, os usuários posicionaram-se entre muito insatisfeito (1) e muito satisfeito (5). $\mathrm{O}$ anexo deste artigo sumariza os itens que compõem o questionário.

Os dados coletados foram organizados, processados e apresentados em tabelas e figuras utilizando-se o programa Statistical Package for the Social Sciences (SPSS 23) para Windows ${ }^{\circledR}$. 
Citação (APA): Santos, J. W. dos, Alsina, O. L. S. de, \& Monteiro, L. F. (2020). Análise da validade e confiabilidade do modelo Servqual adaptado ao varejo supermercadista. Brazilian Journal of Production Engineering, 6(7), 120-132.

\section{RESULTADOS E DISCUSSÃO}

\section{AVALIAÇÃo DA QUALIDADE DOS SERVIÇOS DO SUPERMERCADO}

Na Tabela 2 estão destacados os principais resultados que expressam a expectativa média (E), percepção média (P) e gap médio $(\mathrm{G})$ à luz de cada atributo avaliado pelos clientes do supermercado.

Tabela 2. Síntese da avaliação dos clientes $(\mathrm{n}=385)$

\begin{tabular}{cccccc}
\hline & \multicolumn{2}{c}{ Desempenho $(\mathbf{D})$} & \multicolumn{2}{c}{ Expectativa (E) } & Gap \\
\hline Itens do modelo & Média & Desvio & Média & Desvio & P-E \\
\hline $\mathrm{I}_{1}$ & 3,98 & 0,89 & 4,52 & 0,78 & $-0,54$ \\
$\mathrm{I}_{2}$ & 3,74 & 0,34 & 4,50 & 1,42 & $-0,76$ \\
$\mathrm{I}_{3}$ & 3,41 & 0,78 & 4,91 & 1,68 & $-1,50$ \\
$\mathrm{I}_{4}$ & 4,32 & 1,30 & 4,35 & 1,93 & $-0,03$ \\
$\mathrm{I}_{5}$ & 4,44 & 0,52 & 4,31 & 0,42 & 0,13 \\
$\mathrm{I}_{6}$ & 3,73 & 1,82 & 4,33 & 0,18 & $-0,60$ \\
$\mathrm{I}_{7}$ & 3,68 & 0,77 & 4,29 & 0,22 & $-0,61$ \\
$\mathrm{I}_{8}$ & 2,57 & 0,94 & 4,92 & 0,03 & $-2,35$ \\
$\mathrm{I}_{9}$ & 4,28 & 0,12 & 4,42 & 0,36 & $-0,14$ \\
$\mathrm{I}_{10}$ & 4,40 & 1,56 & 3,71 & 0,18 & 0,69 \\
$\mathrm{I}_{11}$ & 3,90 & 0,78 & 4,50 & 0,33 & $-0,60$ \\
$\mathrm{I}_{12}$ & 4,16 & 1,42 & 4,35 & 0,39 & $-0,19$ \\
$\mathrm{I}_{13}$ & 2,69 & 1,68 & 4,89 & 0,44 & $-2,20$ \\
$\mathrm{I}_{14}$ & 3,37 & 1,93 & 4,85 & 0,31 & $-1,48$ \\
$\mathrm{I}_{15}$ & 4,42 & 1,34 & 4,13 & 0,15 & 0,29 \\
$\mathrm{I}_{16}$ & 4,35 & 0,64 & 4,20 & 0,18 & 0,15 \\
$\mathrm{I}_{17}$ & 4,42 & 0,79 & 4,60 & 0,07 & $-0,18$ \\
$\mathrm{I}_{18}$ & 4,06 & 0,31 & 4,42 & 0,10 & $-0,36$ \\
$\mathrm{I}_{19}$ & 4,37 & 0,77 & 4,43 & 0,12 & $-0,06$ \\
$\mathrm{I}_{20}$ & 3,74 & 0,94 & 4,41 & 0,25 & $-0,67$ \\
$\mathrm{I}_{21}$ & 4,37 & 0,12 & 1,93 & 0,20 & 2,44 \\
$\mathrm{I}_{22}$ & 3,74 & 1,56 & 0,64 & 0,18 & 3,10 \\
\hline
\end{tabular}

Fonte: Autores (2020).

Em relação à avaliação da qualidade dos serviços prestados pelo supermercado, observou-se que os maiores valores médios verificados em relação à expectativa dos clientes foram os itens $\mathrm{I}_{8}(4,92), \mathrm{I}_{3}(4,91), \mathrm{I}_{13}(4,89)$ e $\mathrm{I}_{14}(4,85)$, que correspondem às dimensões confiabilidade, aspectos tangíveis, presteza e segurança, respectivamente. Estes valores demonstram que os clientes possuem uma alta expectativa em relação ao preço dos produtos, as instalações físicas do supermercado, nas diferentes formas de pagamento e no atendimento rápido e eficiente. Observou-se, também, que os maiores valores médios em relação à percepção dos clientes foram os itens $\mathrm{I}_{5}(4,44), \mathrm{I}_{15}(4,42) \mathrm{I}_{17}(4,42)$ e $\mathrm{I}_{10}(4,40)$, que correspondem às dimensões confiabilidade, segurança e presteza, respectivamente. Estes valores demonstram que nestes quesitos os clientes se dizem satisfeitos quanto aos serviços que lhes foram prestados.

Entretanto, percebeu-se que na análise do gap médio, os únicos atributos onde a percepção excedeu às expectativas foram nos itens $\mathrm{I}_{22}(3,10), \mathrm{I}_{21}(2,44), \mathrm{I}_{10}(0,69)$ e $\mathrm{I}_{15}(0,29)$, inseridos nas dimensões empatia, presteza e segurança, respectivamente. Nos demais atributos a maioria dos clientes se dizem insatisfeitos com a qualidade dos serviços, visto que a percepção dos clientes tem sido inferior à expectativa existente, por isto, a maioria dos valores foi negativa. 
Citação (APA): Santos, J. W. dos, Alsina, O. L. S. de, \& Monteiro, L. F. (2020). Análise da validade e confiabilidade do modelo Servqual adaptado ao varejo supermercadista. Brazilian Journal of Production Engineering, 6(7), 120-132.

\section{ANÁliSE DA VALIDADE DO MODELO SERVQUAL ADAPTADO}

Para a análise da validade do modelo, utilizou-se como critério o escore geral do grau de expectativa e desempenho $\left(Y_{i}\right)$. O conjunto das variáveis independentes compreendeu as dimensões: tangibilidade $\left(X_{i 1}\right)$, confiabilidade $\left(X_{i 2}\right)$, presteza $\left(X_{i 3}\right)$, segurança $\left(X_{i 4}\right)$ e empatia $\left(X_{i 5}\right)$. Considerou-se o modelo de regressão linear múltipla, como demonstrado na Equação 3.

$$
Y_{i}=\alpha+\beta 1 X_{i 1}+\beta 2 X_{i 2}+\beta 3 X_{i 3}+\beta 4 X_{i 4}+\beta 5 X_{i 5}+\varepsilon i
$$

A análise de regressão múltipla permitiu inferir sobre a habilidade preditiva do modelo Servqual adaptado em relação ao escore geral do grau de expectativa e desempenho, conforme demostrado na Tabela 3.

Tabela 3. Análise de regressão múltipla do grau de expectativa e desempenho em função das cinco dimensões do modelo $(\mathrm{n}=385)$

\begin{tabular}{lccc|ccc}
\hline & \multicolumn{3}{c|}{ Expectativa (E) } & \multicolumn{3}{c}{ Desempenho (D) } \\
\hline \multicolumn{1}{c}{ Parâmetros } & $\boldsymbol{\beta}$ & Erro-padrão & $\boldsymbol{\beta}$ padronizado & $\boldsymbol{\beta}$ & Erro-padrão & $\boldsymbol{\beta}$ padronizado \\
\hline Constante & $-0,029$ & 0,0054 & - & $-0,046$ & 0,0034 & - \\
Tangibilidade & 0,1487 & 0,0001 & 0,8844 & 0,1539 & 0,0002 & 0,8976 \\
Confiabilidade & 0,1561 & 0,0003 & 0,2989 & 0,1761 & 0,0004 & 0,3749 \\
Presteza & 0,0981 & 0,0007 & 0,5915 & 0,1689 & 0,0009 & 0,7291 \\
Segurança & 0,1088 & 0,0004 & 0,6252 & 0,1974 & 0,0007 & 0,8624 \\
Empatia & 0,1716 & 0,0003 & 0,4541 & 0,1582 & 0,0005 & 0,6131 \\
\hline Coeficiente de determinação & $\mathbf{R = 0 , 8 8 2}$ & $\mathbf{R}^{\mathbf{2}=\mathbf{0 , 7 8 8}}$ & & $\mathbf{R}=\mathbf{0 , 8 9 6}$ & $\mathbf{R}^{\mathbf{2}=\mathbf{0 , 8 0 3}}$ \\
\hline
\end{tabular}

Significância do modelo: $p<0,001$.

Fonte: Autores (2020).

Os coeficientes de determinação ajustados $\left(R^{2}\right)$ foram significativos do ponto de vista estatístico, uma vez que 0,788 das variações do grau de expectativa e 0,803 das variações do grau de desempenho podem ser explicados pela variação dos escores obtidos nas dimensões avaliadas. Como todos os coeficientes apresentaram sinais positivos, a relação entre o escore do grau de satisfação e as demais dimensões é direta, isto é, um aumento nos escores das dimensões implica em um incremento no grau de expectativa e de desempenho.

Considera-se que relações lineares são estatisticamente significativas quando estas apresentam $R^{2}>0,99$ e $p<0,001$. Em termos de validade seria $100 \%$, entretanto esse valor é utópico em se tratando de estudos exploratórios. Assim, os valores de $R^{2}(78,8 \%$ e $80,3 \%)$ são considerados amplamente satisfatório, apontando para a validade do modelo Servqual adaptado (Hair, et al., 2019).

Quando o pressuposto da normalidade dos resíduos é questionado, não existem testes estatísticos específicos para sua avaliação; todavia, os resíduos podem ser plotados com vistas a detecção de sua distribuição próxima a normal e o seu intervalo de variação (Kasznar, \& Gonçalves, 2011). De acordo com Hair, et al., (2019), o gráfico deve apresentar resíduos simétricos em torno de zero. A Figura 1 mostra que a diferença entre os valores observados e estimados pelo modelo de regressão apresentam pequena variação em torno de zero, o que reforça a habilidade preditiva do modelo Servqual adaptado.

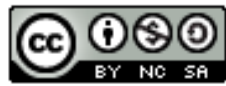



adaptado ao varejo supermercadista. Brazilian Journal of Production Engineering, 6(7), 120-132.

Figura 1. Histograma dos resíduos do modelo de regressão

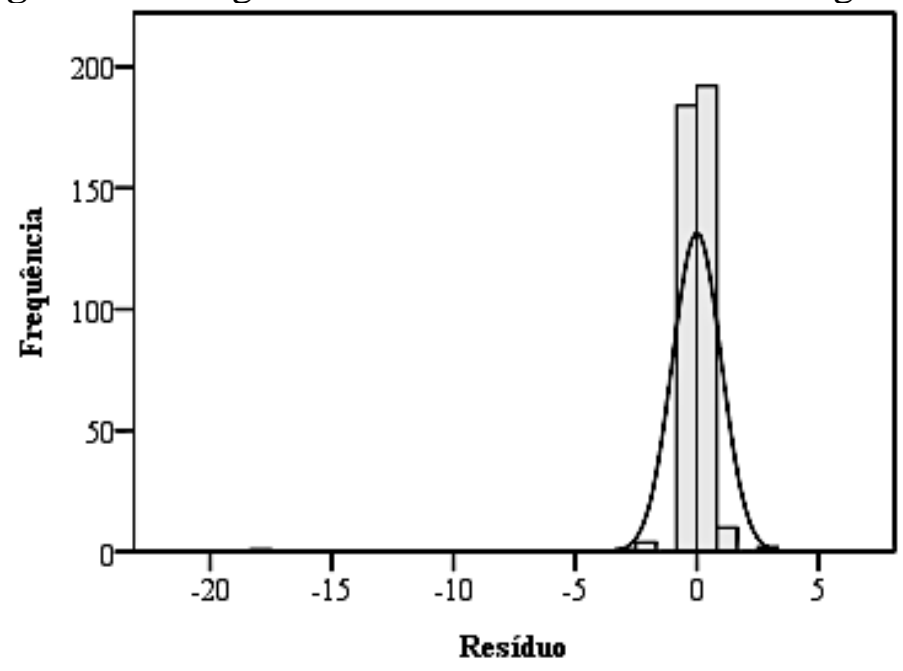

Fonte: Autores (2020).

\section{ANÁLISE DA CONFIABILIDADE DO MODELO SERVQUAL ADAPTADO}

No que concerne à análise da confiabilidade do modelo Servqual adaptado, foram encontrados valores de coeficientes alfa superiores a 0,8 , revelando que a confiabilidade do modelo é considerada alta. Ratifica-se que o valor mínimo aceitável é 0,7 para pesquisas aplicadas e 0,6 para pesquisas exploratórias, conforme preconiza Corrar, et al., (2007). Para a mensuração da expectativa, o valor do alfa foi de 0,87 e para mensuração do desempenho foi de 0,86 . Como o modelo mostrou-se confiável, pode-se concluir que ele efetivamente mediu aquilo o que realmente se propôs de maneira consistente. Porém, depois do estudo preliminar da análise da confiabilidade do modelo, podemos verificar que ao eliminar cada item e realizar o recálculo, o valor do alfa é alterado. Isto é, este coeficiente aumenta ou diminui depois de eliminar um item do modelo.

A Tabela 4 ilustra como os valores de alfa podem ser influenciados quando todos os itens do questionário são considerados em conjunto, bem como o comportamento do coeficiente ao eliminar cada um destes por vez. Na dimensão "Tangibilidade" com a remoção do $\mathrm{I}_{1}$ (25\% do total de itens da dimensão) no questionário que mensura a expectativa, o coeficiente alfa passa de 0,87 para 0,90 , provendo um incremento de $3,45 \%$ na confiabilidade depois da purificação. $\mathrm{O}$ mesmo acontece no questionário que mensura o desempenho, no qual a remoção do $\mathrm{I}_{1}$, colaborou para um incremento de $3,49 \%$ no coeficiente alfa, passando de 0,86 para 0,89 . Portanto, estes itens estão correlacionados de forma menos intensa com os outros itens que constitui o questionário, isto é, as variâncias em suas respostas diminuíam a consistência geral do mesmo, sendo, porém, opcional a eliminação deste item que contribuiu para o aumento do coeficiente alfa. 

adaptado ao varejo supermercadista. Brazilian Journal of Production Engineering, 6(7), 120-132.

Tabela 4. Grau de confiabilidade da consistência interna do questionário

\begin{tabular}{cccc}
\hline & & Expectativa (E) & Desempenho (D) \\
\hline Dimensão & Item & Alfa se item for deletado & Alfa se item for deletado \\
\hline \multirow{4}{*}{ Tangibilidade } & $\mathrm{I}_{1}$ & 0,90 & 0,89 \\
& $\mathrm{I}_{2}$ & 0,87 & 0,85 \\
& $\mathrm{I}_{3}$ & 0,87 & 0,84 \\
& $\mathrm{I}_{4}$ & 0,87 & 0,85 \\
\hline \multirow{5}{*}{ Confiabilidade } & $\mathrm{I}_{5}$ & 0,86 & 0,90 \\
& $\mathrm{I}_{6}$ & 0,89 & 0,84 \\
& $\mathrm{I}_{7}$ & 0,86 & 0,86 \\
& $\mathrm{I}_{8}$ & 0,87 & 0,85 \\
& $\mathrm{I}_{9}$ & 0,88 & 0,90 \\
\hline \multirow{4}{*}{ Presteza } & $\mathrm{I}_{10}$ & 0,85 & 0,86 \\
& $\mathrm{I}_{11}$ & 0,85 & 0,85 \\
& $\mathrm{I}_{12}$ & 0,85 & 0,89 \\
& $\mathrm{I}_{13}$ & 0,89 & 0,85 \\
\hline \multirow{5}{*}{ Segurança } & $\mathrm{I}_{14}$ & 0,86 & 0,85 \\
& $\mathrm{I}_{15}$ & 0,89 & 0,86 \\
& $\mathrm{I}_{16}$ & 0,87 & 0,85 \\
& $\mathrm{I}_{17}$ & 0,89 & 0,89 \\
\hline \multirow{5}{*}{ Empatia } & $\mathrm{I}_{18}$ & 0,87 & 0,85 \\
& $\mathrm{I}_{19}$ & 0,87 & 0,84 \\
& $\mathrm{I}_{20}$ & 0,87 & 0,86 \\
& $\mathrm{I}_{21}$ & 0,87 & 0,86 \\
& $\mathrm{I}_{22}$ & 0,87 & 0,86 \\
\hline Coeficiente alfa de Cronbach & $\mathbf{0 , 8 7}$ & $\mathbf{0 , 8 6}$ \\
\hline
\end{tabular}

Fonte: Autores (2020).

Entretanto, como o nível de alteração do coeficiente alfa foi muito pequeno entre os itens de maior e menor valor aquando das exclusões, optou-se por manter todos os itens no modelo Servqual adaptado.

\section{CONSIDERAÇÕES FINAIS}

O objetivo deste estudo foi analisar a validade e confiabilidade do modelo Servqual operacionalizado em um supermercado da região metropolitana de Aracaju/SE. Com base nos resultados, foi possível perceber que a análise estatística é uma ferramenta importante para as diferentes áreas do conhecimento, não somente como resultado final, mas como um passo ou estágio de uma pesquisa com vistas a investigar o grau de correlação entre as variáveis observadas, para assim, extrair conclusões representativas. Todavia, é importante, conhecer teoricamente os diferentes métodos e as suposições básicas requeridas para a sua utilização de forma adequada.

A análise de regressão linear múltipla e o coeficiente alfa de Cronbach são métodos estatísticos amplamente utilizados para estudar o grau de relacionamento entre variáveis. A investigação desenvolvida para o processo de validação e de confiabilidade realizada com as técnicas supracitadas se mostrou satisfatória, pois os coeficientes encontraram-se acima dos limiares preconizados na literatura especializada.

Além disso, observou-se que a operacionalização do modelo Servqual adaptado gerou algumas implicações gerenciais. A análise dos gaps demonstrou que os itens $\mathrm{I}_{8}(-2,35), \mathrm{I}_{13}$ ($2,20)$, I $I_{3}(-1,50)$ e $I_{14}(-1,40)$ foram os atributos que os consumidores do supermercado possuíam maiores expectativas, mas que apresentaram baixo desempenho. Esses atributos 
Citação (APA): Santos, J. W. dos, Alsina, O. L. S. de, \& Monteiro, L. F. (2020). Análise da validade e confiabilidade do modelo Servqual adaptado ao varejo supermercadista. Brazilian Journal of Production Engineering, 6(7), 120-132.

estavam relacionados, respectivamente, à confiabilidade, presteza, aspectos tangíveis e segurança dos serviços prestados pelo supermercado. Portanto, recomenda-se que esses atributos recebam maior atenção por parte dos gestores, enquanto os pontos fortes sejam utilizados como vantagem competitiva.

É importante destacar que, este estudo apresenta limitações no que se refere à especificidade e tamanho da amostra. Em relação à especificidade da amostra, o estudo conduzido apenas com consumidores cadastrados na base de dados do supermercado pode limitar o modelo de ser utilizado com consumidores que utilizam os serviços de forma eventual. Quanto ao tamanho da amostra, embora o tamanho mínimo estabelecido tenha sido adequado, sugere-se que estudos futuros sejam conduzidos reaplicando o modelo com amostras maiores e diversificada, verificando a aderência do modelo nesse tipo de serviço.

De maneira geral, pode-se observar que a análise estatística foi de grande valia para avaliar critérios que auxiliam na análise dados coletados na pesquisa, pois permitiu uma maior clareza e melhor interpretabilidade dos fenômenos complexos com o qual lidamos; e nesse estudo apresentado, as técnicas empregadas proporcionou verificar, com certa margem de segurança, que o modelo empregado na avaliação da qualidade em serviços do supermercado apresentou-se consistente e preciso.

\section{REFERÊNCIAS}

Adeniran, A. O. (2019). Application of Likert Scale's Type and Cronbach's Alpha Analysis in an Airport Perception Study. Scholar Journal of Applied Sciences and Research, 2(4), 1-5.

Álvarez-García, J., et al., (2019). Quality in Customer Service and Its Relationship with Satisfaction: An Innovation and Competitiveness Tool in Sport and Health Centers. International journal of environmental research and public health, 16(20), 39-42.

Allen, M. J., \& Yen, W. M. (2003). Introduction to measurement theory. WaveLand Press.

Almeida, D., Santos, M. A. R., \& Costa. A. F. B. Aplicação do coeficiente alfa de Cronbach nos resultados de um questionário para avaliação de desempenho da saúde pública. In: XXX Encontro Nacional de Engenharia de Produção, 2010. Anais do...São Paulo. Disponível: http://www.abepro.org.br/biblioteca/enegep2010_TN_STO_131_840_16412.pdf Acesso: 12 out. 2020.

Barbetta, P. A. (2002). Estatística aplicada às ciências sociais. 5. ed. Florianópolis: UFSC.

Bittencourt, H. R., et al., (2011). Desenvolvimento e validação de um instrumento para avaliação de disciplinas na educação superior. Est. Aval. Educação. São Paulo, 22(48) 91114.

Bland, J. M., \& Altman, D. G. (1997). Cronbach’s alpha. British Medical Journal, 19(1), 314572.

Carmines, E. G., \& Zeller, R. A. (1979). Reliability and validity assessment. Sage University paper.

Corrar, L. J.; Paulo, E., \& Dias Filho, J. M. (2007). Análise multivariada. FIPECAFI: Atlas.

Crocker, L., \& Algina, J. (2006). Introduction to classical \& modern test theory. Ed. Thonson, $571 \mathrm{p}$. 
Citação (APA): Santos, J. W. dos, Alsina, O. L. S. de, \& Monteiro, L. F. (2020). Análise da validade e confiabilidade do modelo Servqual adaptado ao varejo supermercadista. Brazilian Journal of Production Engineering, 6(7), 120-132.

Cronbach, L. J. (1951). Coefficient alpha and the internal structure of tests. Psychometrika, 16(3), 297-334.

IBGE (2010). Censo demográficos.

Freitas, A. L. P., \& Rodrigues, S. G. (2005). A avaliação da confiabilidade de questionário: uma análise utilizando o coeficiente alfa de Cronbach. In: XII SIMPEP. Anais do...Bauru, SP.

Freitas, A. L. P., Bolsanello, F. M. C., \& Viana, N. R. N. G. (2008). Avaliação da qualidade de serviços de uma biblioteca universitária: um estudo de caso utilizando o modelo Servqual. Ciência da Informação, 37(3), 88-102.

Freitas, A. L. P., \& Freitas Neto, M. M. (2017). Assessing the service quality in Software-asa-Service from the customers' perspective: a methodological approach and case of use. Production, 27, e20170020.

Freguson, J. M., \& Zawacki, R. A. (1993). Service quality: A critical success factor for IS organizations. Information Strategy: The Executive's Journal, 9(2), 24-30.

Ghotbabadi, A. R., Feiz, S., \& Baharun, R. (2015). Service Quality Measurements: A Review. International Journal of Academic Research in Business and Social Sciences, 5(2), 267-286.

Hair, J. F., et al., (2019). Multivariate data analysis, 8. ed. London, Unites Kingdom: Cengage Learning.

Hair, J. F. (1998). Multivariate data analysis, 5. ed. Englewood Cli's: Prentice-Hall.

Hayes, B. E. (1995). Medindo a satisfação do cliente: desenvolvimento e uso de questionários. Quality Mark, 228 p.

Hora, H. R. M., Monteiro, G. T. R., \& Arica, J. (2010). Confiabilidade em Questionários para Qualidade: Um estudo com o Coeficiente Alfa de Cronbach. Produto \& Produção, 11(2), 85103.

Jan, S., \& Shieh, G. (2019). Sample size calculations for model validation in linear regression analysis. BMC Medical Research Methodology, 19(54), 1-9.

Kasznar, I. K., \& Golçaves, B. M. L. Regressão múltipla: uma digressão sobre seus usos. Disponível: http://www.ibci.com.br/Regressao_Multipla.pdf Acesso: 01 ago 2011.

Kotler, P., \& Armstrong, G. (1998). Princípios de Marketing. Rio de Janeiro: $7^{\mathrm{a}}$ ed. PrenticeAll.

Ko, C. H., \& Chou, C. M. (2020). Apply the SERVQUAL Instrument to Measure Service Quality for the Adaptation of ICT Technologies: A Case Study of Nursing Homes in Taiwan. Healthcare, 8(2), 108-124.

Krus, D. J., \& Helmstadter, G. C. (1993). The problem of negative reliabilities. Educational and Psychological Measurement, 53(1), 643-650.

Malhotra, N. K. (2012). Pesquisa de Marketing. $6^{\text {a }}$ ed. Bookman.

Nichols, D. P. My Coefficient $\alpha$ is Negative, SPSS Keywords. Disponível: http://www.ats.ucla.edu/STAT/SPSS/library/negalfa.htm Acesso: 20 set. 2020.

Parasuraman, A., Zeithaml, V. A., \& Berry, L. L. (1985). A conceptual model of service quality and its implications for future research. Journal of Marketing, 49(1), 41-50.

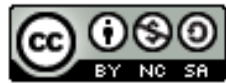



adaptado ao varejo supermercadista. Brazilian Journal of Production Engineering, 6(7), 120-132.

Parasuraman, A., Zeithaml, V. A., \& Berry, L. L. (1990). An empirical test of the extended gaps model of service quality. Marketing Science Institute, 1(1), 90-122.

Bolarinwa, O. A. (2015). Principles and methods of validity and reliability testing of questionnaires used in social and health science researches. Niger Postgraduate Medical Journal, 22(1), 195-201.

Roy, S., Sreejesh, S., \& Bhatia. (2019). S. Service quality versus service experience: An empirical examination of the consequential effects in B2B services. Industrial Marketing Management, 82(1), 52-69.

Santos, J. W. (2016). Proposição de um modelo multifatorial para mensuração da satisfação de consumidores do varejo supermercadista. Revista Gestão Industrial, 12(3), 198-215.

Santos, J. W. Um modelo multifatorial para medir a satisfação dos consumidores de supermercados. In: Simpósio de engenharia de produção de Sergipe, 2016. Anais do... São Cristóvão.

Disponível: https://ri.ufs.br/bitstream/riufs/7696/2/ModeloMultifatorialSupermercados.pdf Acesso: 24 de set. 2020.

Santos, J. W., Gomes, K. T. S., \& Vasconcelos, C. R. Aplicação de métodos de controle de estoque no varejo supermercadista. In: Simpósio de engenharia de produção de Sergipe, 2017. Anais do... São Cristóvão. Disponível: http://simprod.ufs.br/pagina/21037 Acesso: 25 set. 2020.

Santos, J. W., et al., Avaliação da qualidade dos serviços prestados em um supermercado sob à ótica do cliente: uma abordagem exploratória. In: Simpósio de engenharia de produção de Sergipe, 2015. Anais do... São Cristóvão. Disponível: https://ri.ufs.br/bitstream/riufs/8270/2/QualidadeServicosSupermecadoCliente.pdf Acesso: 25 set. 2020.

Schneider, B., \& White, S. (2004). Service quality: Research perspectives. California: Sage Publications.

Streiner, D. L. (2003). Being inconsistent about consistency: when coefficient alpha does and doesn't matter. Journal of Personality Assessment, 80(1), 217-222.

Silva, E. L., \& Menezes, E. M. (2001). Metodologia da pesquisa e elaboração de dissertação. Florianópolis.

Taber, K. S. (2018). The Use of Cronbach's Alpha When Developing and Reporting Research Instruments in Science Education. Research in Science Education, 48(1), 1273-1296. 